Received: 10 January 2019

Accepted: 27 June 2019

Published online: 07 August 2019

\section{SNARE protein SEC22B regulates early embryonic development}

Shin-Rong J. Wu ${ }^{1,2}{ }^{2}$, Rami Khoriaty ${ }^{3}$, Stephanie H. Kim ${ }^{1,2}$, K. Sue O'Shea ${ }^{4}$, Guojing Zhu ${ }^{5}$, Mark Hoenerhoff ${ }^{6}$, Cynthia Zajac $\mathbb{B}^{3}$, Katherine Oravecz-Wilson ${ }^{3}$, Tomomi Toubai ${ }^{3}$, Yaping Sun ${ }^{3}$, David Ginsburg ${ }^{3,5,7,8}$ \& Pavan Reddy ${ }^{1,3}$

The highly conserved SNARE protein SEC22B mediates diverse and critical functions, including phagocytosis, cell growth, autophagy, and protein secretion. However, these characterizations have thus far been limited to in vitro work. Here, we expand our understanding of the role Sec22b plays in vivo. We utilized Cre-Lox mice to delete Sec22b in three tissue compartments. With a germline deletion of $\operatorname{Sec} 22 b$, we observed embryonic death at E8.5. Hematopoietic/endothelial cell deletion of Sec22b also resulted in in utero death. Notably, mice with Sec22b deletion in CD11c-expressing cells of the hematopoietic system survive to adulthood. These data demonstrate $\operatorname{Sec} 22 b$ contributes to early embryogenesis through activity both in hematopoietic/endothelial tissues as well as in other tissues yet to be defined.

Intracellular trafficking plays a critical role in cellular biology, regulating the distribution and organization of secretory proteins. One protein class which helps mediate this complex choreography is the SNAREs (soluble NSF attachment protein receptor). Partner SNAREs bind and mediate the fusion of two membranes by physically bringing the membranes sufficiently close to fuse ${ }^{1}$. SEC22B is an endoplasmic reticulum (ER)-SNARE which localizes to the ER and the ER-Golgi intermediate compartment ${ }^{2}$. It functions as a vesicular-SNARE ${ }^{3,4}$ and an R-SNARE engaged in antero- and retrograde ER-Golgi transport ${ }^{5,6}$. Its known interacting partners are varied. It forms a classic four-helix SNARE complex with Qa-SNARE syntaxin 18, Qb-SNARE BNIP1, and Qc-SNARE p31/Use1 at the ER membrane ${ }^{7}$. SEC22B is also known to interact with plasma membrane/Qa-SNAREs syntaxin $1^{8}$, syntaxin $4^{2}$ and syntaxin $5^{4}$, suggesting it also functions at the interface between the plasma membrane and the ER membrane.

At the cellular level, in addition to its role in ER-Golgi trafficking, SEC22B appears to mediate membrane expansion under several conditions, including Legionella and Leishmania infection in macrophages ${ }^{9-11}$ as well as during axonal growth from isolated cortical neurons ${ }^{8}$. Some evidence suggests that SEC22B contributes to cellular homeostasis as well. For example, in murine macrophages, SEC22B negatively regulates phagocytosis ${ }^{12}$ but $^{-}$ promotes reactive oxygen species accumulation during S. aureus infection ${ }^{13}$. In flies, Sec22 influences ER morphology ${ }^{14}$, while in yeast, Sec22 contributes to autophagosome biogenesis ${ }^{15}$. In human cell lines, SEC22B has been implicated in the secretory autophagy pathway ${ }^{16}$ as well as in macroautophagy ${ }^{17}$. SEC22B also contributes to other secretory pathways, such as that in VLDL (very-low-density lipoprotein)-secreting rat hepatocytes. Thus, current evidence suggests that $\operatorname{Sec} 22 b$ is highly conserved and plays a fundamental role in cell biology. However, while cDNA library-based expression profiling has demonstrated that $\operatorname{Sec} 22 b$ is expressed in murine embryos ${ }^{18-20}$, its function in embryogenesis in vivo remains unexplored.

Utilizing Cre-Lox mice, we deleted Sec22b from all tissues, from hematopoietic and endothelial cells, and from CD11c-expressing cells, a subset of the hematopoietic cell population. We observed that $\operatorname{Sec} 22 b$ is critical for embryonic development. Embryos with a global deficiency in $\operatorname{Sec} 22 b$ do not survive beyond 8.5 days post coitum (E8.5). Furthermore, deletion of Sec $22 b$ from the hematopoietic compartment with Vav1-Cre results in embryonic lethality. However, normal development was observed with deletion of Sec $22 b$ in CD11c-expressing hematopoietic cells.

\footnotetext{
${ }^{1}$ Program in Immunology, University of Michigan Medical School, Ann Arbor, USA. ${ }^{2}$ Medical Scientist Training Program, University of Michigan Medical School, Ann Arbor, USA. ${ }^{3}$ Department of Internal Medicine, Michigan Medicine, Ann Arbor, USA. " Department of Cellular and Developmental Biology, University of Michigan Medical School, Ann Arbor, USA. '5ife Sciences Institute, University of Michigan, Ann Arbor, USA. ${ }^{6}$ Unit for Laboratory Animal Medicine, University of Michigan, Ann Arbor, USA. ${ }^{7}$ Department of Human Genetics, University of Michigan Medical School, Ann Arbor, USA. ${ }^{8}$ Howard Hughes Medical Institute, University of Michigan, Ann Arbor, USA. Correspondence and requests for materials should be addressed to P.R. (email: reddypr@med.umich.edu)
} 


\section{Results}

Sec22b is necessary for embryonic development. To determine the role of SEC22B in vivo, we intercrossed mice heterozygous for a FRT recombination site-flanked conditional gene-trapped Sec22b allele $\left(\operatorname{Sec} 22 b^{\text {tmlal+ }}\right)$ (Fig. 1a,b), but did not detect any Sec22 $b^{\text {tm laltmla }}$ offspring at weaning $(p<0.0001)$ (Table 1). To exclude the possibility that an off target gene trap effect ${ }^{21}$, as opposed to the loss of functional Sec $22 b$, was responsible for this phenotype, we generated mice heterozygous for the $\operatorname{Sec} 22 b$ null allele $\left(\operatorname{Sec} 22 b^{+l-}\right)$. First, we crossed the $\operatorname{Sec} 22 b^{\text {tm1a }}$ allele to mice expressing FLP recombinase driven by the human $\beta$-actin promoter, and excised the gene trap cassette, resulting in the Sec $22 b^{f l}$ allele (Fig. 1a,c), where exon 3 is flanked by LoxP sites (Fig. 1a). Subsequently, the $\operatorname{Sec} 22 b^{f t}$ allele was crossed to mice expressing Cre recombinase driven by the germline-expressed EIIa promoter, producing the Sec $22 b^{-}$allele (Fig. 1a,d). Sec $22 b^{+/-}$mice exhibited normal survival $(p=0.6473)$ (Table 1). However, no Sec22 $b^{-1-}$ pups were observed at weaning $(p=0.0008)$ (Table 1).

Sec22 $b^{-I-}$ mice do not survive beyond E8.5. To determine the stage at which germline loss of $\operatorname{Sec} 22 b$ results in embryonic death, we next performed timed matings on Sec $22 b^{+1-}$ intercrosses. Offspring from this intercross exhibited Mendelian genotypic distribution at E3.5 $(p=0.6929)$ and E7.5 $(p=0.4685)$ (Table 1). However, at E8.5, $\mathrm{Sec} 22 b^{-1-}$ mice were significantly underrepresented $(p=0.0055)$ (Table 1). Thereafter, at E9.5, E11.5, and E13.5, no Sec22 $b^{-1-}$ embryos were observed (Table 1). Thus, Sec $22 b^{-1-}$ embryos do not survive beyond E8.5.

Loss of Sec22b does not impact embryo size at E7.5. To attempt to investigate the mechanism driving the lethality we observed at E8.5 of Sec22 $b^{-1-}$ embryos, we examined Sec $22 b^{-1-}$ embryos one day earlier, at E7.5. At this stage, Sec $22 b^{+/-}$and Sec $22 b^{-1-}$ embryos appeared smaller than Sec $22 b^{+/+}$ones (Fig. 2a), though this trend did not reach statistical significance (Fig. 2b). Notably, some Sec $22 b^{-1-}$ embryos showed a developmental delay, appearing to be in the egg cylinder stage as opposed to the early somite stage (Fig. 2a).

We hypothesized the impact of Sec $22 b$ heterozygosity on size required additional time to reach significance. Because Sec $22 b^{-1-}$ mice die in utero, to address this, we compared weight of Sec $22 b^{+1-}$ adult mice to littermate control wildtype mice. However, we did not observe a difference in size, suggesting that $\operatorname{Sec} 22 b$ heterozygosity does not impact growth (Fig. 2c).

Vav1-Cre driven deficiency of Sec22b results in embryonic lethality. SEC22B has a known role in immune cell function, particularly in mediating intracellular transport in myeloid cells $\mathrm{s}^{2,9-13,16}$. Interestingly, defects in intracellular transport are known to cause lysosomal storage disorders in humans, which result in early mortality and notably can be treated with bone marrow transplantation ${ }^{22,23}$. Thus, we hypothesized that loss of $\operatorname{Sec} 22 b$ in the hematopoietic compartment would lead to embryonic lethality. To test this, we used Vav1-Cre to drive deletion of $\operatorname{Sec} 22 b$ in hematopoietic tissue. While Vav1-Cre also exhibits variable activity between $8-70 \%$ in endothelial cells, depending on anatomical location ${ }^{24}$, its excisional efficiency in endothelial tissue remains lower than that in hematopoietic cells ${ }^{25}$.

Using a Sec22 $b^{f l+}$; Vav1-Cre ${ }^{+} \times \operatorname{Sec} 22 b^{f l f f}$; Vav1-Cre $e^{-}$breeding scheme, we observed a significantly reduced number of Sec22 $b^{f l f f}$; Vav1-Cre mice at weaning $(p<0.0001)$ (Table 2). Additionally, we observed a trend towards fewer Sec22bflff; Vav1-Cre ${ }^{+}$and Sec22 $b^{f l-}$; Vav1-Cre ${ }^{+}$embryos at E12.5 (Table 2), one day after Vav1 expression begins ${ }^{26,27}$. To understand how loss of Sec22b in hematopoietic cells and endothelial cells might be causing embryonic lethality, we examined an E12.5 Sec $22 b^{f /-}-V^{f} 1-C r e^{+}$embryo for evidence of dysfunction in hematopoietic organs where hematopoietic stem cells are found. Interestingly, in the liver, we observed enlarged endothelial-lined hepatic sinusoids and binucleate erythroid progenitors (Fig. 3).

Finally, while some Vav1-Cre-mediated knockout embryos survived to weaning (Table 2), amplification of genomic DNA at the $\operatorname{Sec} 22 b$ locus in peripheral blood cells from these survivors (Fig. 1e) suggests that incomplete excision at exon 3 of $\operatorname{Sec} 22 b$ may explain survival to weaning and into adulthood (Fig. 4a).

Sec22 $b^{+l-}$ mice exhibit no hematopoietic phenotype under physiologic conditions. Because hematopoietic loss of $\operatorname{Sec} 22 b$ results in embryonic lethality (Table 2), we wondered if $\operatorname{Sec} 22 b^{+/-}$mice might exhibit a hematopoietic phenotype. To test this, we performed complete blood counts (CBCs) on peripheral blood collected from Sec $22 b^{+/-}$mice. These were indistinguishable from that obtained from littermate controls, including total white blood cells, monocytes, lymphocytes, neutrophils, hemoglobin, and platelet counts (Fig. 5).

Mice with Sec22b deletion in CD11 $c^{+}$cells survive to adulthood. Given the embryonic lethality observed with Vav1-mediated deletion of Sec22b, the known role for SEC22B in macrophages s $^{10-13}$ and dendritic cells $(\mathrm{DCs})^{2}$ in vitro, and the role of intracellular transport in lysosomal storage disorders ${ }^{23}$, we hypothesized loss of $\operatorname{Sec} 22 b$ in a myeloid cell population would result in embryonic lethality. To test this, we used Itgax-Cre (CD11c-Cre) to delete Sec22b in CD11c-expressing bone-marrow derived DCs (BMDCs) (Sec22b fl/f; CD11c-Cre ${ }^{+}$) (Fig. 4b,c). To our surprise, offspring generated by crossing Sec22 $b^{f l+}$; $\mathrm{CD} 11 \mathrm{c}-\mathrm{Cr} e^{+}$and $\mathrm{Sec} 22 \mathrm{~b}^{\mathrm{flf} f}$; CD11c-Cre ${ }^{-}$ mice exhibited the expected Mendelian distribution (Table 3) as well as normal survival up to 6 months (168 days) (Fig. 4d).

\section{Discussion}

Our studies demonstrate that $\operatorname{Sec} 22 b$ is required in vivo for survival past E8.5 (Table 1). While we observed normal development of $\operatorname{Sec} 22 b$ heterozygous adult mice (Fig. 2c), some null embryos demonstrated a developmental delay at E7.5 (Fig. 2a,b), though this did not reach statistical significance when assessed by embryonic length (Fig. 2b). Thus, the mechanism by which $\operatorname{Sec} 22 b$ is required for in vivo survival remains undefined.

Our data additionally demonstrate that deletion of $\operatorname{Sec} 22 b$ from the hematopoietic compartment also results in partial embryonic lethality (Table 2) and may cause abnormal erythropoiesis, based on preliminary histologic 
a
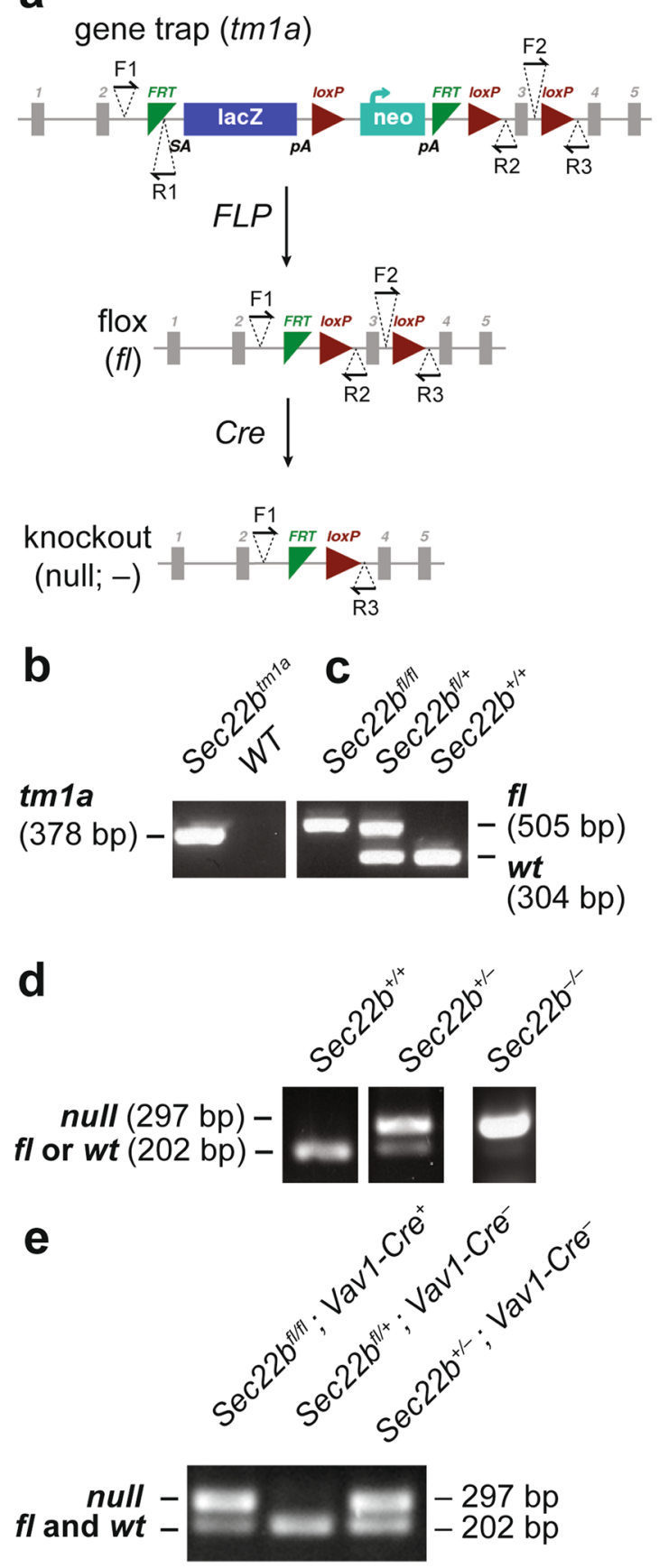

Figure 1. Generation of gene targeted $\operatorname{Sec} 22 b$ alleles. (a) $\operatorname{Sec} 22 b$-conditional gene trapped mice (tm $1 a$ ), with a FRT-flanked gene trap inserted between exons 2 and 3 were mated to FLP-recombinase transgenic mice to create floxed $(f l)$ mice, with LoxP sites flanking exon 3. Floxed mice were mated to EIIa-Cre to generate the germline null allele $\left(\operatorname{Sec} 22 b^{-}\right)$, or to Vav1-Cre or CD11c-Cre transgenic mice to generate tissue specific Sec $22 b$ deficiency. Binding sites for genotyping primers (F1, F2, R1, R2, R3) are indicated with half arrowheads. (b) PCR with primers F1 and R1 detects the insertion of the conditional gene trap in Sec $22 b(t m 1 a)$. (c) PCR with primers F1 and R2 detects the excision of the conditional gene trap by FLP recombinase and distinguishes between $\operatorname{Sec} 22 b^{f l}$ homozygous and heterozygous mice. (d) Competitive PCR with primers F1, F2, and R3 detects the excision of exon 3 of $\operatorname{Sec} 22 b$ and distinguishes between $\operatorname{Sec} 22 b^{-}$heterozygous and homozygous mice. (e) PCR on genomic DNA isolated from peripheral blood of a surviving Sec22b $b^{f l f l}$; Vav1-Cre ${ }^{+}$mouse and a $\mathrm{Sec} 22 \mathrm{~b}^{f l+}$; Vav1-Cre $e^{-}$littermate control, compared to DNA from Sec $22 b^{+l-}$ mice. Gel images (b-e) are cropped. Full-length images may be found in Supplementary Fig. S1a-c.

data (Fig. 3a). Further work is necessary to characterize the impact of Sec $22 b$ on embryonic hematopoiesis. Notably, Sec $22 b$ heterozygosity does not cause a hematopoietic phenotype under physiologic conditions (Fig. 5) and deletion of $\operatorname{Sec} 22 b$ within a specific hematopoietic subpopulation, CD11c-expressing cells, did not reproduce 


\begin{tabular}{|c|c|c|c|c|}
\hline a. Genotype: & $\operatorname{Sec} 22 b^{+1+}$ & $\operatorname{Sec} 22 b^{t m 1 a /+}$ & $\operatorname{Sec} 22 b^{t m I a / t m I a}$ & $p$-value \\
\hline $\begin{array}{l}\text { Sec22b } b^{\text {tmIa/+}} \times \operatorname{Sec} 22 b^{t m 1 a /+} \text { Expected } \\
\text { Ratios }\end{array}$ & $25 \%$ & $50 \%$ & $25 \%$ & \\
\hline At weaning $(n=73)$ & $33 \%(24)$ & $67 \%(49)$ & $0 \%(0)$ & $<0.0001$ \\
\hline b. Genotype: & ${\operatorname{Sec} 22 \mathbf{b}^{+/+}}^{+1}$ & ${\operatorname{Sec} 22 b^{+/-}}$ & & p-value \\
\hline $\operatorname{Sec} 22 b^{+/-} \times \operatorname{Sec} 22 b^{+/+}$Expected Ratios & $50 \%$ & $50 \%$ & & \\
\hline Weaning $(\mathrm{n}=234)$ & $48 \%(113)$ & $52 \%(121)$ & & 0.3237 \\
\hline c. Genotype: & $\operatorname{Sec} 22 b^{+/+}$ & $\operatorname{Sec} 22 b^{+/-}$ & $\operatorname{Sec} 22 b^{-1-}$ & $p$-value \\
\hline $\operatorname{Sec} 22 b^{+l-} \times \operatorname{Sec} 22 b^{+/-}$Expected Ratios & $25 \%$ & $50 \%$ & $25 \%$ & \\
\hline Weaning $(\mathrm{n}=34)$ & $24 \%(8)$ & $76 \%(26)$ & $0 \%(0)$ & $<0.0001$ \\
\hline $\mathrm{E} 13.5(\mathrm{n}=9)$ & $22 \%(2)$ & $78 \%(7)$ & $0 \%(0)$ & 0.0751 \\
\hline $\mathrm{E} 11.5(\mathrm{n}=21)$ & $24 \%(5)$ & $76 \%(16)$ & $0 \%(0)$ & 0.0024 \\
\hline E9.5 $(n=20)$ & $25 \%(5)$ & $75 \%(15)$ & $0 \%(0)$ & 0.0032 \\
\hline $\mathrm{E} 8.5(\mathrm{n}=35)$ & $34 \%(12)$ & $60 \%(21)$ & $6 \%(2)$ & 0.0033 \\
\hline E7.5 $(n=23)$ & $13 \%(3)$ & $65 \%(15)$ & $22 \%(5)$ & 0.4685 \\
\hline $\mathrm{E} 3.5(\mathrm{n}=33)$ & $27 \%(9)$ & $52 \%(17)$ & $21 \%(7)$ & 0.3938 \\
\hline
\end{tabular}

Table 1. Genotypic distribution of offspring from $\operatorname{Sec} 22 b^{\text {tm } 1 a /+}$ and $\operatorname{Sec} 22 b^{+/-}$mating schemes. (a) Genotypic distribution of offspring at weaning from Sec22 $b^{\text {tm } 1 a /+}$ intercrosses with expected Mendelian distribution. (b) Genotypic distribution of offspring at weaning from $\operatorname{Sec} 22 b^{+/-} \times \operatorname{Sec} 22 b^{+/+}$crosses compared to expected Mendelian distribution. (c) Genotypic distribution of offspring at weaning and at indicated days post coitum (e.g. E13.5) from Sec22 $b^{+/-}$intercrosses as compared to expected Mendelian distribution. $P$-values are calculated from a one-tailed binomial test for (a, c) $\operatorname{Sec} 22 b^{-1-}$ and for (b) $\operatorname{Sec} 22 b^{+/-}$versus all other genotypes.

a
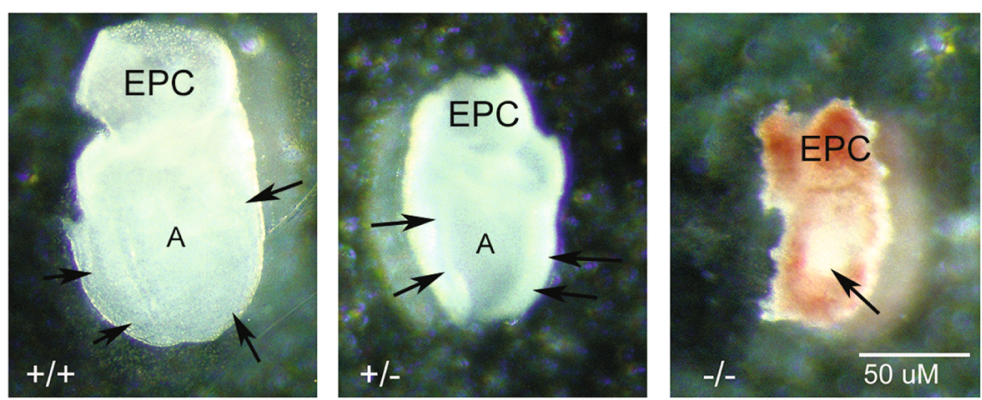

b
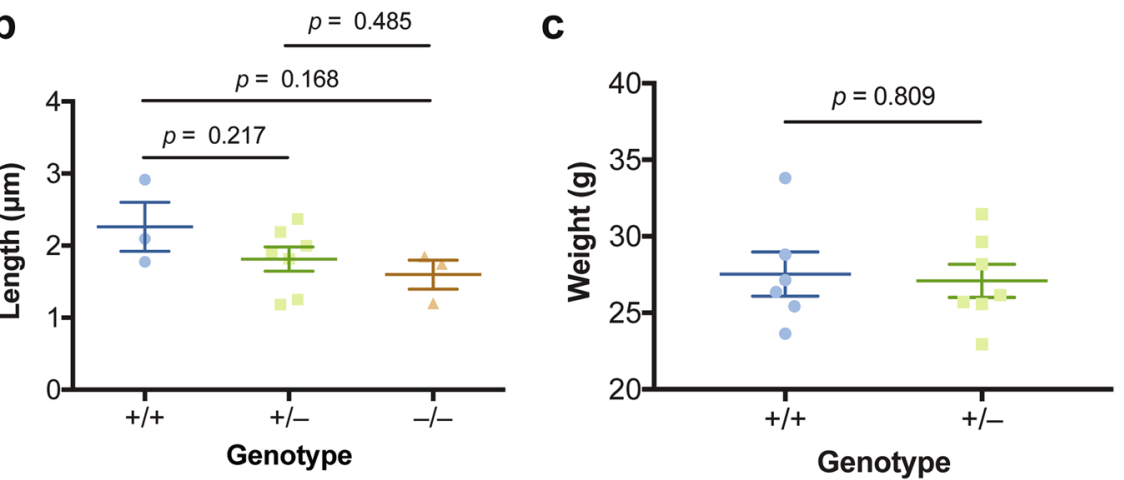

Figure 2. Characterization of $\operatorname{Sec} 22 b^{+/-}$and $\operatorname{Sec} 22 b^{-1-}$ mice. (a) Lateral views of embryos from the three genotypes. The ectoplacental cone (EPC) is located at the dorsal surface of the embryos. The $+/+\left(\operatorname{Sec} 22 b^{+/+}\right)$ embryo is beginning to convert to a primitive streak staged embryo, and is characterized by a well defined ectoderm (arrows) surrounding the expanded amniotic cavity, while the $+/-\left(\operatorname{Sec} 22 b^{+/-}\right)$embryo is at the late egg cylinder stage with a clear ectoderm layer and expanding amniotic cavity. The $-/-\left(\operatorname{Sec} 22 b^{-/-}\right)$embryo is surrounded by decidua and Reichert's membrane, the ectoderm is just beginning to elongate to form the egg cylinder (arrow). (b) Length $(\mu \mathrm{m})$ comparison between $\operatorname{Sec} 22 b^{+1+}(\mathrm{WT}, n=3), \operatorname{Sec} 22 b^{+1-}(\mathrm{Het}, n=7)$, $\operatorname{Sec} 22 b^{-1-}(\mathrm{KO}, n=3)$ embryos using an unpaired two-tailed $t$ test. Error bars represent SEM. (c) Weight in grams of 4.5-5 month old $\operatorname{Sec} 22 b^{+1-}(\mathrm{n}=7)$ and $\operatorname{Sec} 22 b^{+/+}$littermate controls $(\mathrm{n}=6)$ mice using an unpaired two-tailed $t$ test. Error bars represent SEM. 


\begin{tabular}{|c|c|c|c|c|c|}
\hline a. Genotype: & Sec $22 b^{f l f l} ;$ Vav1-Cre $e^{-}$ & Sec $22 b^{f l /+} ;$ Vav1-Cre $e^{-}$ & $\operatorname{Sec} 22 b^{f l /+} ;$ Vav1-Cre $e^{+}$ & $\begin{array}{l}\text { Sec22b } b^{f l / f} \\
\text { Vav1-Cre }\end{array}$ & $p$-value \\
\hline $\begin{array}{l}\text { Sec22b } b^{f l /+} ; \text { Vav1-Cre } e^{+} \times \operatorname{Sec} 22 b^{f l f l} ; \operatorname{Vav1-} \\
\text { Cre }^{-} \text {Expected Ratios }\end{array}$ & $25 \%$ & $25 \%$ & $25 \%$ & $25 \%$ & \\
\hline Observed at weaning $(\mathrm{n}=177)$ & $29 \%(52)$ & $29 \%(52)$ & $36 \%(64)$ & $5 \%(9)$ & $<0.0001$ \\
\hline b. Genotype: & $\operatorname{Sec} 22 b^{f l / f l} ; \operatorname{Vav1-Cre^{+}}$ & $\operatorname{Sec} 22 b^{f l /-} ; \operatorname{Vav1-Cre^{+}}$ & All other genotypes & & \\
\hline $\begin{array}{l}\text { 1. Sec22b fll+; Vav1-Cre }{ }^{+} \times \operatorname{Sec}^{f l 2} b^{f l-} ; \text { Vav1- } \\
\text { Cre }^{-} \text {Expected Ratios }\end{array}$ & $12.5 \%$ & $12.5 \%$ & $75 \%$ & & \\
\hline $\mathrm{E} 12.5(\mathrm{n}=18)$ & $0 \%(0)$ & $11.1 \%(2)$ & $88.9 \%(16)$ & & 0.1353 \\
\hline $\begin{array}{l}\text { 2. Sec22b }{ }^{+/-} ; \text {Vav1-Cre } e^{+} \times \operatorname{Sec} 22 b^{f l f f} ; \text { Vav1- } \\
\text { Cre }^{-} \text {Expected Ratios }\end{array}$ & $12.5 \%$ & $12.5 \%$ & $75 \%$ & & \\
\hline $\operatorname{E} 12.5(n=19)$ & $0 \%(0)$ & $3.0 \%(1)$ & $97.0 \%(18)$ & & 0.0310 \\
\hline
\end{tabular}

Table 2. Genotypic distribution of offspring from Sec22 $b^{f l+} ; \mathrm{Vav}_{1-C r} e^{+} \times \operatorname{Sec} 22 b^{f l f l}$; Vav1-Cre $e^{-}$mating pairs. (a) Genotypic distribution of offspring at weaning from Sec $22 b^{f l+}$; Vav1-Cre ${ }^{+} \times S e c 22 b^{f l f l}$; Vav1-Cre $e^{-}$crosses as compared to expected Mendelian distribution. (b) Genotypic distribution of offspring at E12.5 from (1) Sec22 $b^{f l+}$; Vav1-Cre $\times$ Sec22 $b^{f l-}$; Vav1-Cre $e^{-}$and (2) Sec22 $b^{+1-}$; Vav1-Cre ${ }^{+} \times \operatorname{Sec} 22 b^{f l f l}$; Vav1-Cre $e^{-}$crosses as compared to expected Mendelian distribution. $P$-values for (a) are calculated from a one-tailed binomial test for Sec $22 b^{f l l f l}$; Vav1-Cre ${ }^{+}$versus all other genotypes. $P$-values for (b) are calculated from a one-tailed binomial test comparing the knockout embryos (both Sec22b ${ }^{f l f l}$; Vav1-Cre ${ }^{+}$and $\operatorname{Sec} 22 b^{f l-}$; Vav1-Cre ${ }^{+}$) versus all other genotypes.

the embryonic lethality (Table 3, Fig. 4d). Interestingly, loss of Sec22b in this compartment does not seem to affect the development or function of $\mathrm{CD} 11 \mathrm{c}^{+}$cells $^{28}$.

Vav1-Cre also exhibits recombination in endothelial cells, albeit less efficiently than in hematopoietic cells ${ }^{24}$. Thus it is possible that loss of $\operatorname{Sec} 22 b$ in endothelial cells may also drive lethality. Whether this is through their function as blood vessel endothelium or as progenitors of hematopoietic stem cells ${ }^{29}$ must be investigated in the future. The significance of altered hepatic sinusoidal morphology (Fig. 3) remains unclear. Interestingly, a recent study described a patient exhibiting growth delay, intellectual disability, hepatopathy, joint contracture and immunodeficiency with multiple homozygous recessive mutations, including in $\operatorname{Sec} 22 b^{30}$.

Taken together, our data suggest $\operatorname{Sec} 22 b$ is necessary in at least two cell compartments for embryonic survival: Vav1-expressing and non-expressing cells. Vav1 transcripts are first detectable at E11.5 $26,27,31$. By E12.5, there were reduced populations of Sec22 $2^{f l / f l}$; Vav1-Cre ${ }^{+}$and Sec22 $b^{f l-}$; Vav1-Cre ${ }^{+}$embryos (Table 2), which reached significance by weaning (Table 2), demonstrating the necessity of Sec22b expression in $\mathrm{Vav1}^{+}$cells for embryonic survival. Furthermore, given that global deletion of $\operatorname{Sec} 22 b$ results in embryonic lethality by E8.5 (Table 1), Sec22b expression in another tissue compartment is also necessary for embryonic survival.

This Vav1-non-expressing cell compartment in which $\operatorname{Sec} 22 b$ expression is required for embryonic survival remains undefined. The loss of Sec $22 b^{-1-}$ embryos at E8.5 could be due to the absence of Sec $22 b$ in the first wave of embryonic blood cells, which arise in the fetal yolk sac at E7.25 $5^{32}$, or in another cell compartment. Sec $22 b$ has also previously been shown to be required in vitro for axonal growth in isolated mouse cortical neurons ${ }^{8}$ and for VLDL secretion in rat hepatocytes ${ }^{33}$, while in D. melanogaster, loss of Sec22 resulted in defects in eye development $^{14}$. Furthermore, mutations to a SEC22B partner SNARE, GOSR2, have been associated with progressive myoclonic epilepsy ${ }^{5}$. Finally, SEC22B has also been implicated in pancreatic $\beta$-cell proinsulin secreation ${ }^{34}$. Future studies may explore how $\operatorname{Sec} 22 b$ expression in these tissues alters embryonic development and survival and may facilitate the development of clinically translatable models.

\section{Methods}

Mice. Mice with a FRT-flanked conditional gene trap inserted between exons 2 and 3 of Sec $22 b$ were obtained from the European Conditional Mouse Mutagenesis Program (EUCOMM; Sec22 $b^{\mathrm{tm} l \mathrm{a}(\mathrm{EUCOMM}) \mathrm{Wtsi}}$ ) and crossed to mice with FLP recombinase expressed under the control of human $\beta$-actin promoter (005703, The Jackson Laboratory) to create the Sec22 $b^{f l}$ allele. EIIa-Cre (003724, The Jackson Laboratory), Vav1-Cre (008610, The Jackson Laboratory), and CD11c-Cre transgenic mice (008068, The Jackson Laboratory), were bred to $\operatorname{Sec} 22 b^{f l / f l}$ mice to create $\operatorname{Sec} 22 b^{f l f l}$; EIIa-Cre ${ }^{+}, \operatorname{Sec} 22 b^{f l f l}$; Vav1-Cre ${ }^{+}, \operatorname{Sec} 22 b^{f l f l}$; CD11c-Cre $e^{+}$mice. Mice acquired from Jackson Laboratory had been backcrossed to the C57/BL6 background as described in their catalog. Mice acquired from EUCOMM were generated using the C57/BL6 background. All animals were cared for under regulations reviewed and approved by the University of Michigan Institutional Animal Care and Use Committee, based on University Laboratory Animal Medicine guidelines.

Timed matings. Breeding pairs were co-housed in the evening. Females were checked for the presence of a vaginal plug the following morning ( 0.5 days post coitum; E0.5). Those with plugs were tracked and euthanized at the appropriate time point and embryos were dissected from uteri and, where indicated, photographed prior to fixation.

DNA Isolation. Weaned mice and adult mice were genotyped by digesting tail clips in $200 \mathrm{uL} /$ tail clip DirectPCR Lysis Reagent (Mouse Tail) (Viagen, 102-T) and $4 \mathrm{uL} /$ tail clip Proteinase K (Sigma Aldrich, P4850) at $56^{\circ} \mathrm{C}$ overnight followed by denaturing at $95^{\circ} \mathrm{C}$ for 1 hour. Genomic DNA from embryos aged E7.5-13.5 and from peripheral blood was obtained using the DNeasy Blood \& Tissue Kit (Qiagen, 69504), following manufacturer's 

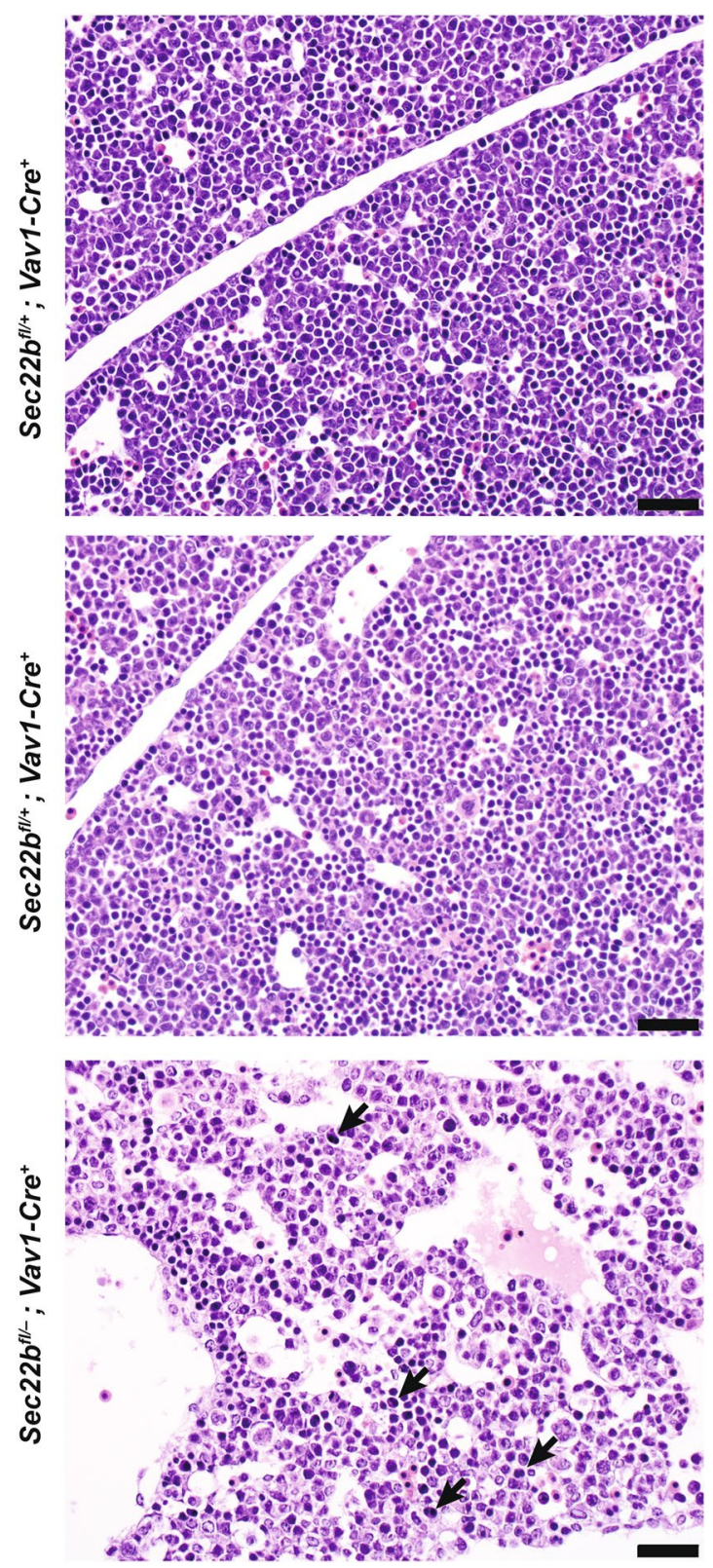

Figure 3. Histopathologic characterization of Vav1-Cre mediated excision at E12.5. H\&E visualization of E12.5 liver from Sec22b $b^{f l-}$ Vav1-Cre $e^{+}$and Sec22 $b^{f l+}$ Vav1-Cre embryos with arrows indicating binucleate erythroid progenitors. Size bars all represent $20 \mu \mathrm{m}$.

instructions. E3.5 blastocysts were harvested into 1xPBS into PCR tubes (USA Scientific, 1402-2500), frozen at $-80^{\circ} \mathrm{C}$, and then thawed. Thawed product was used for genotyping PCR.

Primers and genotyping. Primers used to genotype Cre transgenes and the $\operatorname{Sec} 22 b$ allele are collected in Table 4. Binding locations for the Sec $22 b$ primers are identified in Fig. 1a. The Sec $22 b$ F1 + R1 primers was used to identify gene-trapped mice and the $\operatorname{Sec} 22 b \mathrm{~F} 1+\mathrm{R} 2$ primers was used to identify floxed versus wildtype mice. Sec22b F1, F2, R3 were used in a competitive PCR to identify the null allele versus the floxed allele. Genotyping was performed via PCR reaction with GoTaq Green Master Mix (Promega, M7122) according to manufacturer's recommendations.

Gels were imaged using AlphaImager HP. Resulting images were processed using AutoContrast.

Imaging and analysis. Embryos were dissected and imaged by light microscopy (Leica, DM IRB). ImageJ was used to calculate the surface area of photographed embryos. Images were prepared with Adobe Photoshop CS6, using the AutoContrast and AutoTone features and by setting the gamma correction to 0.5 . 
a

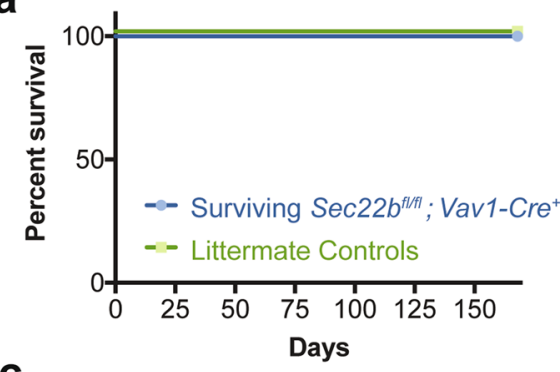

C

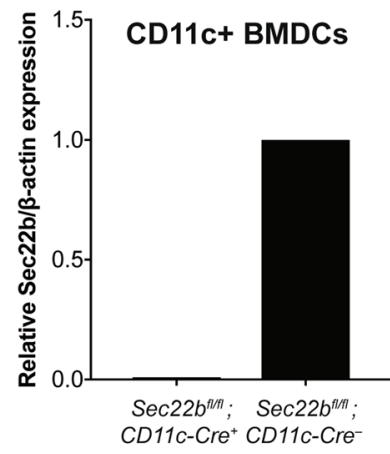

Genotypes b CD11c+ BMDCs

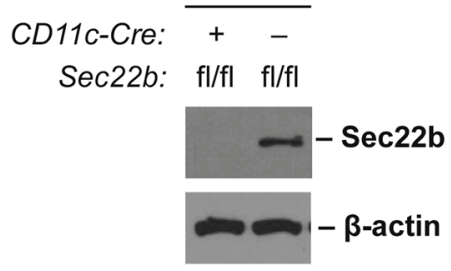

d

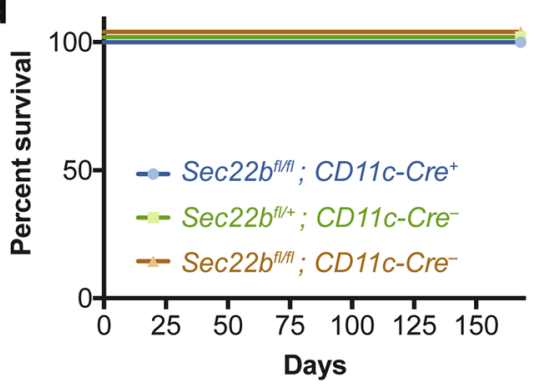

Figure 4. Survival of mice with $\operatorname{Sec} 22 b$ deletion in hematopoietic subsets. (a) 6 month survival curve for surviving Sec $22 b^{f l / f l}$; Vav1-Cre $e^{+}$mice $(n=5)$ as compared to littermates $(n=4)$. (b) Western Blot and (c) corresponding quantification of CD11c + MACS-sorted BMDCs from Sec $22 b^{f l f f}$; CD11c-Cre $e^{+}$mice and $\mathrm{Sec} 22 \mathrm{~b}^{f l f f}$; CD11c-Cre ${ }^{-}$. The Western Blot image (b) is cropped. Full-length images may be found in Supplementary Fig. S1d. (d) 6 month survival curve for Sec $22 b^{\text {fllfl }}$; CD11c-Cre ${ }^{+}$mice $(\mathrm{n}=53)$ as compared to $\operatorname{Sec} 22 b^{f l+} ; C D 11 c-C r e^{-}(\mathrm{n}=52)$ and $\operatorname{Sec} 22 b^{f l / f l} ; C_{11} c-C_{r}^{-}(\mathrm{n}=38)$ littermates.
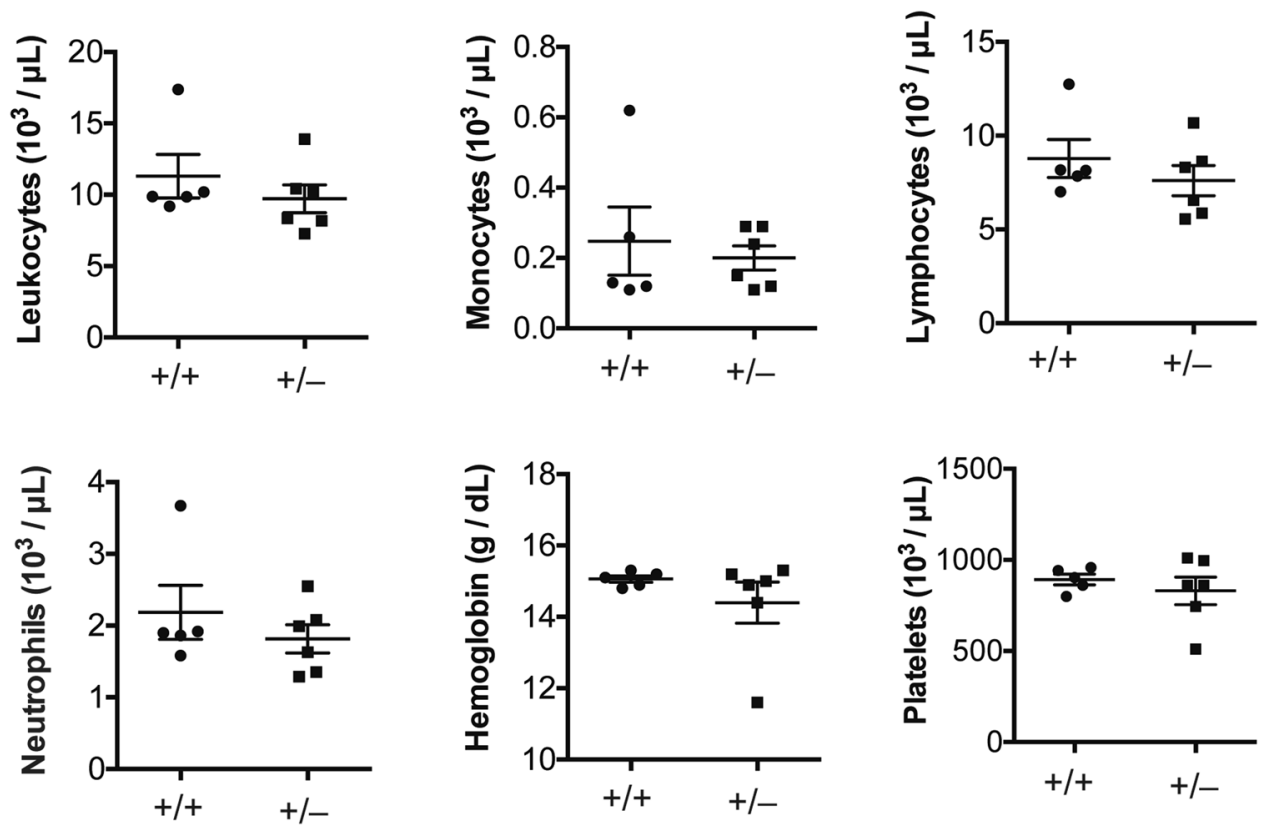

Figure 5. Complete blood counts on $\operatorname{Sec} 22 b$ heterozygous mice. Total leukocytes, monocytes, lymphocytes, neutrophils, hemoglobin, and platelets from peripheral blood of 5 month old $\operatorname{Sec} 22 b^{+/+}(\mathrm{n}=5)$ and $\operatorname{Sec} 22 b^{+/-}$ $(\mathrm{n}=6)$ littermates quantified as indicated on axes compared using unpaired two-tailed t tests. Error bars represent SEM. 


\begin{tabular}{|c|c|c|c|c|c|}
\hline Genotype: & $\begin{array}{l}\text { Sec22b } b^{f l f l} ; \\
\text { CD11c-Cre }\end{array}$ & $\begin{array}{l}\text { Sec } 22 b^{f l++} \\
\text { CD11c-Cre }\end{array}$ & $\begin{array}{l}\text { Sec } 22 b^{f l /+} \\
\text { CD11c-Cre }\end{array}$ & $\begin{array}{l}\text { Sec22b flffl; } \\
\text { CD11c-Cre }\end{array}$ & $p$-value \\
\hline Sec22b $b^{f l /+} ; C D 11 c-C r e^{+} \times S e c 22 b^{f l f l} ; C D 11 c-C r e^{-}$Expected Ratios & $25 \%$ & $25 \%$ & $25 \%$ & $25 \%$ & \\
\hline Observed at weaning $(n=606)$ & $28 \%(171)$ & $25 \%(149)$ & $22 \%(133)$ & $25 \%(153)$ & 0.4596 \\
\hline
\end{tabular}

Table 3. Genotypic distribution of offspring from Sec $22 b^{f l+} ; C D 11 c-C r e^{+} \times S e c 22 b^{f l f l} ; C D 11 c-C r e^{-}$mating pairs. Genotypic distribution of offspring at weaning from Sec $22 b^{f l+} ; C D 11 c-C r e^{+} \times S e c 22 b^{f l f f}$; CD11c-Cre mating pairs as compared to expected Mendelian distribution. $P$-values are calculated from a one-tailed binomial test for $\operatorname{Sec} 22 b^{f l f l}$; CD11c-Cre $e^{+}$versus all other genotypes.

\begin{tabular}{|l|l|}
\hline Primer Name & Sequence $\left(\mathbf{5}^{\prime} \rightarrow \mathbf{3}^{\prime}\right)$ \\
\hline Sec $22 b \mathrm{~F} 1$ & AAGGGTGGATGGATTCTTCACAC \\
\hline Sec $22 b \mathrm{~F} 2$ & TCCTTTTGAATGGAGAAAGCTTC \\
\hline Sec2 $2 b$ R 1 & TTGGTGGCCTGTCCCTCTCACCTT \\
\hline Sec2 $2 b$ R 2 & GCAGCTCAGCAGTAAGAACACGTC \\
\hline Sec2 $2 b$ R 3 & CCTGTGACAGTCTACAGATTGGA \\
\hline Cre $\mathrm{F}$ & TTACCGGTCGATGCAACGAGT \\
\hline Cre $\mathrm{R}$ & TTCCATGAGTGAACGAACCTGG \\
\hline Vav1 $\mathrm{F} 1$ & AGATGCCAGGACATCAGGAACCTG \\
\hline Vav1 R1 & ATCAGCCACACCAGACACAGAGATC \\
\hline Vav1 22 & CTAGGCCACAGAATTGAAAGATCT \\
\hline Vav1 R2 & GTAGGTGGAAATTCTAGCATCATC \\
\hline
\end{tabular}

Table 4. Primers used for Sec22b and Cre genotyping. Sec22b primers include F1 and F2 forward primers and $\mathrm{R} 1, \mathrm{R} 2$, and R3 reverse primers. Sec22b primer binding positions are indicated in Fig. 1a. Cre primers detect both EIIa- and CD11c-Cre transgenes. Used together, Vav1 primers detect the Vav1-Cre transgene.

Histologic methods. E12.5 embryos were embedded in OCT compound and frozen. Sections were then placed on glass slides and stained with hematoxylin and eosin.

Western blot. Whole cell lysates were obtained and protein concentrations determined by BCA Protein Assay (Thermo Scientific, 23225). Protein was separated by SDS-PAGE gel electrophoresis and transferred to PVDF membrane (Millipore, IPVH00010) using a Bio-Rad semi-dry transfer cell (1703940) (20 V, $1 \mathrm{~h})$. Blots were incubated with SEC22B (1:200, Santa Cruz, 29-F7) and b-Actin (1:1000, abcam, ab8226) primary antibodies overnight at $4{ }^{\circ} \mathrm{C}$. Incubation with secondary anti-mouse antibody conjugated to HRP $(1: 10,000$, Santa Cruz, sc-2005) was performed for 1 hour at room temperature. Bound antibody was revealed using SuperSignal ECL substrate (Thermo Scientific). Conversion to grayscale and densitometric analysis was performed using ImageJ.

Complete blood counts. Peripheral blood was collected into K2 EDTA-coated Microvette collection tubes (Sarstedt, 16.444.100). CBCs were performed with a HEMAVet950 (Drew Scientific, CT) at the University of Michigan In Vivo Animal Core.

Statistics. All statistical analysis was performed using Graphpad Prism 7. Specific tests are indicated in the respective figure legends.

\section{Data Availability}

All data generated or analysed during this study are included in this published article or in Supplementary Information.

\section{References}

1. Chen, Y. A. \& Scheller, R. H. SNARE-mediated membrane fusion. Nat Rev Mol Cell Biol 2, 98-106, https://doi.org/10.1038/35052017 (2001).

2. Cebrian, I. et al. Sec22b regulates phagosomal maturation and antigen crosspresentation by dendritic cells. Cell 147, 1355-1368, https://doi.org/10.1016/j.cell.2011.11.021 (2011).

3. Zhang, T., Wong, S. H., Tang, B. L., Xu, Y. \& Hong, W. Morphological and functional association of Sec22b/ERS-24 with the preGolgi intermediate compartment. Mol Biol Cell 10, 435-453, https://doi.org/10.1091/mbc.10.2.435 (1999).

4. Xu, D., Joglekar, A. P., Williams, A. L. \& Hay, J. C. Subunit structure of a mammalian ER/Golgi SNARE complex. J Biol Chem 275, 39631-39639, https://doi.org/10.1074/jbc.M007684200 (2000).

5. Volker, J. M. et al. Functional assays for the assessment of the pathogenicity of variants of GOSR2, an ER-to-Golgi SNARE involved in progressive myoclonus epilepsies. Dis Model Mech 10, 1391-1398, https://doi.org/10.1242/dmm.029132 (2017).

6. Garcia-Castillo, M. D. et al. Retrograde transport is not required for cytosolic translocation of the B-subunit of Shiga toxin. JCell Sci 128, 2373-2387, https://doi.org/10.1242/jcs.169383 (2015).

7. Aoki, T., Kojima, M., Tani, K. \& Tagaya, M. Sec22b-dependent assembly of endoplasmic reticulum Q-SNARE proteins. Biochem J 410, 93-100, https://doi.org/10.1042/BJ20071304 (2008).

8. Petkovic, M. et al. The SNARE Sec22b has a non-fusogenic function in plasma membrane expansion. Nature cell biology advance on, https://doi.org/10.1038/ncb2937 (2014). 
9. Arasaki, K. \& Roy, C. R. Legionella pneumophila promotes functional interactions between plasma membrane syntaxins and Sec22b. Traffic 11, 587-600, https://doi.org/10.1111/j.1600-0854.2010.01050.x (2010).

10. Arasaki, K., Toomre, D. K. \& Roy, C. R. The Legionella pneumophila effector DrrA is sufficient to stimulate SNARE-dependent membrane fusion. Cell Host Microbe 11, 46-57, https://doi.org/10.1016/j.chom.2011.11.009 (2012).

11. Canton, J., Ndjamen, B., Hatsuzawa, K. \& Kima, P. E. Disruption of the fusion of Leishmania parasitophorous vacuoles with ER vesicles results in the control of the infection. Cell Microbiol 14, 937-948, https://doi.org/10.1111/j.1462-5822.2012.01767.x (2012).

12. Hatsuzawa, K. et al. Sec22b is a negative regulator of phagocytosis in macrophages. Mol Biol Cell 20, 4435-4443, https://doi. org/10.1091/mbc.E09-03-0241 (2009).

13. Abuaita, B. H., Burkholder, K. M., Boles, B. R. \& O’Riordan, M. X. The Endoplasmic Reticulum Stress Sensor Inositol-Requiring Enzyme 1alpha Augments Bacterial Killing through Sustained Oxidant Production. MBio 6, e00705, https://doi.org/10.1128/ mBio.00705-15 (2015).

14. Zhao, X. et al. Sec22 regulates endoplasmic reticulum morphology but not autophagy and is required for eye development in Drosophila. J Biol Chem 290, 7943-7951, https://doi.org/10.1074/jbc.M115.640920 (2015).

15. Nair, U. et al. SNARE proteins are required for macroautophagy. Cell 146, 290-302, https://doi.org/10.1016/j.cell.2011.06.022 (2011).

16. Kimura, T. et al. Dedicated SNAREs and specialized TRIM cargo receptors mediate secretory autophagy. EMBO J36, 42-60, https:// doi.org/10.15252/embj.201695081 (2017).

17. Renna, M. et al. Autophagic substrate clearance requires activity of the syntaxin-5 SNARE complex. J Cell Sci 124, 469-482, https:// doi.org/10.1242/jcs.076489 (2011).

18. Ko, M. S. et al. Large-scale cDNA analysis reveals phased gene expression patterns during preimplantation mouse development. Development 127, 1737-1749 (2000).

19. Carninci, P. et al. The transcriptional landscape of the mammalian genome. Science 309, 1559-1563, https://doi.org/10.1126/ science.1112014 (2005)

20. Hay, J. C., Chao, D. S., Kuo, C. S. \& Scheller, R. H. Protein Interactions Regulating Vesicle Transport between the Endoplasmic Reticulum and Golgi Apparatus in Mammalian Cells. Cell 89, 149-158, https://doi.org/10.1016/S0092-8674(00)80191-9 (1997).

21. Maguire, S. et al. Targeting of Slc25a21 is associated with orofacial defects and otitis media due to disrupted expression of a neighbouring gene. PLoS One 9, e91807, https://doi.org/10.1371/journal.pone.0091807 (2014).

22. Hoogerbrugge, P. M. et al. Allogeneic bone marrow transplantation for lysosomal storage diseases. The European Group for Bone Marrow Transplantation. Lancet 345, 1398-1402 (1995).

23. Platt, F. M. Sphingolipid lysosomal storage disorders. Nature 510, 68-75, https://doi.org/10.1038/nature13476 (2014).

24. Inra, C. N. et al. A perisinusoidal niche for extramedullary haematopoiesis in the spleen. Nature 527, 466-471, https://doi. org/10.1038/nature15530 (2015).

25. de Boer, J. et al. Transgenic mice with hematopoietic and lymphoid specific expression of Cre. Eur J Immunol 33, 314-325, https:// doi.org/10.1002/immu.200310005 (2003).

26. Zmuidzinas, A. et al. The vav proto-oncogene is required early in embryogenesis but not for hematopoietic development in vitro. ЕМВО J 14, 1-11 (1995).

27. Bustelo, X. R., Rubin, S. D., Suen, K. L., Carrasco, D. \& Barbacid, M. Developmental expression of the vav protooncogene. Cell Growth Differ 4, 297-308 (1993).

28. Wu, S. J. et al. A Critical Analysis of the Role of SNARE Protein SEC22B in Antigen Cross-Presentation. Cell Rep 19, 2645-2656, https://doi.org/10.1016/j.celrep.2017.06.013 (2017).

29. Eilken, H. M., Nishikawa, S. \& Schroeder, T. Continuous single-cell imaging of blood generation from haemogenic endothelium. Nature 457, 896-900, https://doi.org/10.1038/nature07760 (2009).

30. Diao, H., Zhu, P., Dai, Y. \& Chen, W. Identification of 11 potentially relevant gene mutations involved in growth retardation, intellectual disability, joint contracture, and hepatopathy. Medicine (Baltimore) 97, e13117, https://doi.org/10.1097/ MD.0000000000013117 (2018).

31. Keller, G., Kennedy, M., Papayannopoulou, T. \& Wiles, M. V. Hematopoietic commitment during embryonic stem cell differentiation in culture. Mol Cell Biol 13, 473-486 (1993).

32. Palis, J., Robertson, S., Kennedy, M., Wall, C. \& Keller, G. Development of erythroid and myeloid progenitors in the yolk sac and embryo proper of the mouse. Development 126, 5073-5084 (1999).

33. Siddiqi, S., Mani, A. M. \& Siddiqi, S. A. The identification of the SNARE complex required for the fusion of VLDL-transport vesicle with hepatic cis-Golgi. Biochem J 429, 391-401, https://doi.org/10.1042/BJ20100336 (2010).

34. Fan, J. et al. cTAGE5 deletion in pancreatic beta cells impairs proinsulin trafficking and insulin biogenesis in mice. J Cell Biol 216 , 4153-4164, https://doi.org/10.1083/jcb.201705027 (2017).

\section{Acknowledgements}

This work was supported by the US National Institutes of Health (NIH) grants HL-128046, HL-090775, CA173878, CA-203542, R35-HL135793, K08 HL128794, and the Herman and Dorothy Miller Fund. We acknowledge the In Vivo Animal Core at the University of Michigan for their technical expertise. Rami Khoriaty is a recipient of the American Society of Hematology Scholar Award. David Ginsburg is a Howard Hughes Medical Institute Investigator.

\section{Author Contributions}

Conceptualization, S.J.W., R.K., K.S.O., D.G., P.R.; Methodology, S.J.W., R.K., K.S.O., G.Z., M.H. Validation, S.J.W., R.K., S.H.K., K.S.O., G.Z., M.H. Formal analysis, S.J.W., K.S.O., M.H. Investigation, S.J.W., R.K., S.H.K., K.S.O., G.Z., M.H., C.Z., K.O.W., T.T., Y.S. Resources, R.K., D.G. Writing-Original Draft, S.J.W. WritingReview \& Editing, S.J.W., R.K., K.S.O., M.H., D.G., P.R. Visualization, S.J.W. Supervision, R.K., D.G., P.R. Funding Acquisition, S.J.W., P.R.

Additional Information

Supplementary information accompanies this paper at https://doi.org/10.1038/s41598-019-46536-7.

Competing Interests: The authors declare no competing interests.

Publisher's note: Springer Nature remains neutral with regard to jurisdictional claims in published maps and institutional affiliations. 
(c) (i) Open Access This article is licensed under a Creative Commons Attribution 4.0 International License, which permits use, sharing, adaptation, distribution and reproduction in any medium or format, as long as you give appropriate credit to the original author(s) and the source, provide a link to the Creative Commons license, and indicate if changes were made. The images or other third party material in this article are included in the article's Creative Commons license, unless indicated otherwise in a credit line to the material. If material is not included in the article's Creative Commons license and your intended use is not permitted by statutory regulation or exceeds the permitted use, you will need to obtain permission directly from the copyright holder. To view a copy of this license, visit http://creativecommons.org/licenses/by/4.0/.

(C) The Author(s) 2019 